\title{
Reflection through Gaming: Reinforcing health message response through gamified rehearsal
}

\author{
Piiastiina Tikka*, Miia Laitinen, Iikka Manninen, and Harri Oinas-Kukkonen \\ University of Oulu, Pentti-Kaiteran katu 1, Oulu, Finland
}

\begin{abstract}
Reflection is generally considered an effective means of achieving behavior change. A gamified approach to promoting rehearsal and reflection in a healthy eating context was studied. The game was based on the principles of the Implicit Attitude Test: by categorizing food items under positive or negative associations the players would gain points according to how fast they categorized foods under positive or negative associations. Game scores constituted feedback for reflection, and repeated playing constituted rehearsal of target responses. Experiment participants $(\mathrm{N}=58)$ played the game over a five-day period. Constructs of Rehearsal (REH), self-reported questionnaire responses on Reflection (REFL) and Perceived Persuasiveness (PEPE), and self-reported Perceived Health Behavior Change (PHBC) were analyzed using PLS-SEM. The results show that PLAY moderates the REFL-PEPE relationship, and there are also significant relationships between REH and PEPE, PEPE and PHBC, and REFL and PHBC.
\end{abstract}

Keywords: Persuasive technology, Behavior change, Gamification, Selfreflection, Perceived persuasiveness, PSD, PLS-SEM

\section{Introduction}

The role of reflection in behavior change is well established [1, 2, 3] and typically the approach is to allow system users to monitor their own behavior - a common device for such self-monitoring being activity bracelets or smart watches. A plethora of sensors carried by modern information technology products ensures that there is no shortage of means for monitoring what people do physically, but how can technology support turning that monitoring into actual reflection and, in turn, potential for behavior change? What mechanisms are required in order to help turn reflection into action?

In health behavior, monitoring behavior provides a good view of whether a person is doing what he or she is meant to do: a food diary (provided it is accurate) will reveal if the diet is what it should be, or an activity record from a bracelet will show if there has been enough activity and exercise in a day. It is also known that the attrition rates with systems for behavior change is rather high [4]. To even start using such a system a person first needs to become aware of a problem. Further to that, acknowledging a problem is only the first step, while learning and developing ways of addressing it is quite another. Activity monitoring or food diaries are good methods for 
tracking behavior and a source for feedback, which a person can use as the basis for reflection. They can be, however, overwhelming in the active effort required.

In the present study we wanted to explore the potential of gamifying a method of feedback provision on decision-making and even implicit attitudes as means of supporting a change in existing thinking. Gaming has been associated with rehearsal [5, 6] and combining a rehearsal with an immediate feedback through the engagement in a game offers an appealing environment for learning and reinforcing target behaviors. We developed a mobile game 'Implicity' based on the principles of the Implicit Association Test (IAT) [7] where the prevailing implicit associations and attitudes are presumed to be automatic and reactions to relevant stimuli are faster than to stimuli opposite to the automatic attitudes. We hypothesized that by playing a game where the player has to quickly categorize food items into positive and negative categories would a) expose the player to a possible attitude bias in their thinking, and b) through repetition the player would be rehearsing responses to types of food as if learning by rote. For example, when the player is consistently slower in placing vegetables into the positive category than placing bacon in the negative category, the message to the player would be that they need to be aware of their automatic choices and preferences, and by playing the game more the player can rehearse and learn the target response. The reflection that takes place can teach the player to observe his or her own thinking as regards target behavior. Health behavior in the present study is vegetable and fruit consumption.

In the present study we then ask 1) does a gamified process of drawing attention to implicit attitudes evoke self-reflection, 2) does gamification of response rehearsal contribute positively to behavior change?

\section{Background}

The theoretical cornerstones for the present study are found in the Behavior Change Support System (BCSS) framework and the Persuasive Systems Design (PSD) model $[8,9]$. Building on this base we used gamification principles [10] and the Implicit Association Test (IAT) [7] to create a game mechanism, feedback provisioning (for reflection) and engagement elements in the form of a highly gamified BCSS.

\subsection{Persuasive Systems Design and Behavior Change Support Systems}

A system that from the onset aims at behavioral and psychological outcomes, but does this openly and without coercion or deception is, by definition, a Behavior Change Support System (BCSS) [8]. The aims and goals to be defined when developing a behavior change support system involve the type and expected outcome of the system: should the system form, reinforce or change a user's compliance, behavior or attitude? Further to that, the Persuasive Systems Design (PSD) model is a tool for analyzing and implementing those goals through system features [9].

The development of the game and the selection of persuasive system features was guided by a PSD analysis of the goals, intentions and persuasion context. The main goals of the system were identified as changing behavior and/or attitude, or reinforc- 
ing behavior and/or attitude where change was not necessary. Gamification aspects of the system used Dialogue Support features praise and rewards. More at the core of the persuasiveness were Primary Task Support features of rehearsal, self-monitoring and reduction. System credibility support features included reliable sources for health information, transparency in sources, and use of authorities. Of the core persuasive features the role of rehearsal should be highlighted: playing a game can involve numerous repetitions of the set tasks, which in the present case means numerous repetitions of quick decision-making as regards food items. Such repetition means that the target response is rehearsed at a quick pace in volumes that a person would not readily encounter in real life. A player of such a game also rehearses the target response.

Many persuasive systems are built to encourage reflection (cf. especially selfmonitoring in the PSD model) that is expected to lead to change in behaviors while others engage in a more prescriptive approach [1]. Open-ended reflection can, perhaps, be seen as a less obtrusive means to an end than a purposefully prescriptive one. The PSD model [9] postulates that one of the key elements of a persuasive system is that it is unobtrusive: it does not get in the way of a system user's primary task. Reflection, when a by-product of an activity, can be seen as a subtle approach to paying attention to behaviors and to changing them. The feedback from the game allows the player to observe his or her own thinking in a game context.

\subsection{Gamification}

Using core characteristics of gaming (self-purposefulness and hedonistic use) with an ultimately utilitarian goal such as behavior change or learning is the essence of gamification [10]. Gamification repurposes the intrinsic motivation that goes with game play as a tool for utilitarian use by using typical game elements of points, badges, leaderboards, goals, narratives, feedback and achievements in the system design [10, 11]. At the highly gamified end the utilitarian benefits come almost as a side product of a pleasurable activity. Just as in BCSS development, gamifying a system or a service involves definitions of goals [12]. Target behavior is analyzed and the system is set to monitor the defined performance, then features supporting engagement and fun (a key part of any game) are developed [12]. On the one hand, gamification of behavior change could be seen as a form of "sugaring the pill", but on the other hand the appeal of the gamification approach may be more closely related to the pursuit of unobtrusiveness. Using gamification as an element of a BCSS is not unheard of, and it can be done successfully $[13,14,15,16,17]$. Further to that, cognitive tests have also been used before as the basis of a gamified system [18].

\subsection{Implicit Association Test}

The IAT [7] was developed to test implicit attitudes by revealing automatic associations. In the test subjects' response times to test objects are used to determine automatic associations. While in the present study the developed game does not follow the IAT concept fully so that it could be used to determine a player's implicit associations as such, the concept of reaction times as an indicator of automatic associations is used as the basic mechanism for scoring and game progression. In research IAT has been used also in healthy eating and diet related studies. For example, people who actively 
avoided high-calorie foods also showed an implicit association with low-calorie foods [19], and obese people showed stronger negative implicit associations towards highfat foods than a control group [20]. In the present paper the focus is on the effect of rehearsal, and the IAT scores over the test period are not included. This selection was necessary in order to limit the scope of the present paper.

\section{$3 \quad$ Method}

\subsection{Model, hypotheses, and measurement instruments}

The research model (Fig 1) illustrates the constructs of Reflection (REFL), Rehearsal (REH), Perceived Persuasiveness (PEPE), and Perceived Health Behavior Change (PHBC) and their relationships, as hypothesized. In addition, a further hypothesis (H8) is presented regarding the effect of the study on reported fruit and vegetable consumption. Measurement instruments used in the study are described in Table 5.

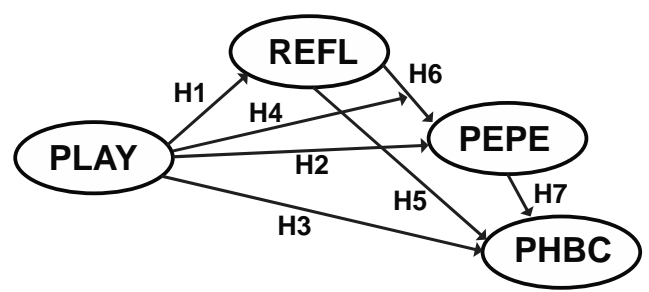

Figure 1. Research model and hypotheses.

Rehearsal (REH) is the number of game sets played by each participant. The PSD model [9] presents rehearsal as a means in a system for a user to rehearse a behavior. Participants were asked to play a minimum of three sets per day on five consecutive days, and they were told that they were free to play as much as they wished. The more an individual plays the game the more he or she is exposed to the underlying health message and the feedback (score) on his or her (implicit) associations. We hypothesize that the exposure will lead to reflection, higher perceived persuasiveness of the system, as well as have a positive effect on post-experiment health behavior. We also hypothesize that REH has a moderating effect on the relationship between REFL and PEPE.

Table 1. Hypotheses regarding REH construct.

$H$ Higher number of game sets played means a higher level of exposure to the health message, leading to a positive effect on Reflection.

Higher number of game sets played offers a higher level of repetition of the target response and it

H2 directs a player to associate healthier foods with positive words (or vice versa). Such exposure will have a positive effect of Rehearsal on Perceived Persuasiveness of the system.

Higher number of game sets will directly enforce the concept of healthier food choices in a re-

H3 peated way as the player is expected to categorize foods and words. The volume of game play will thus have a positive effect on the Perceived Health Behavior Change. 
Reflection (REFL) construct indicates engagement in reflection [21], how individuals inspect and evaluate their own thoughts, feelings and behaviors [22]. While H1 supposes that playing a game would trigger users to think about their thinking more, it is likely this tendency is already present in some degree in the participants. However, regardless of its origin, reflection is a component in measuring an individual's readiness for purposeful behavior change [21].

Table 2. Hypotheses regarding REFL construct.

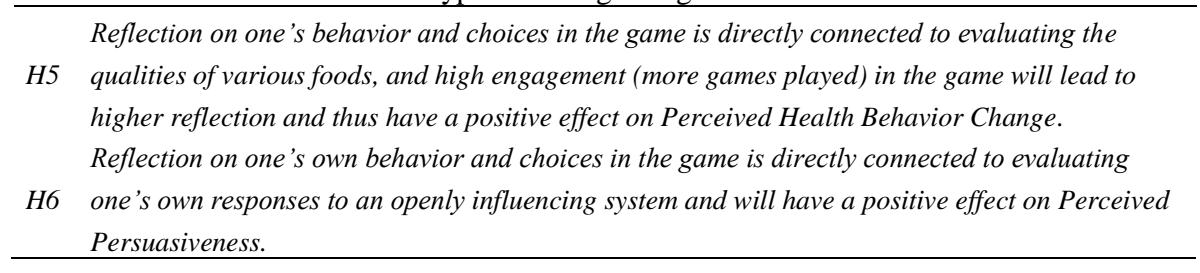

Perceived Persuasiveness (PEPE) is a construct combining system user's perception of the system itself and its message into an attitude item that consists of cognitions and affect $[23,24]$. A favorable attitude and a person's assessment of the system and its effect on his or her own behavior or thinking is a factor in a system's ability to support behavior change [24, 25].

Table 3. Hypotheses regarding Perceived Persuasiveness construct.

Perceived Persuasiveness of a system has a positive effect on the readiness of an individual to

H7 immediately evaluate the direct impact of the system on his or her behavior, and supports system users in engaging in target behavior.

Perceived Health Behavior Change (PHBC) is a construct made of open-ended evaluations by participants on the actual changes they may have noticed after the experiment. The construct is close to PEPE in that it is based on an individual's perception of behavior change impact as regards the system. However, PHBC assesses the direct result of system use, in the present case increased use of fruit and vegetables or contemplation of existing dietary habits with a view to engage in behavior change. Where PEPE as an instrument focuses on evaluating how the user relates to the system and on the respondent's view of the role of the system in any change and personal relevance, the PHBC instrument asks the participants in an open-ended question to describe the exact and actual change (or lack of it). In addition to the PHBC as a measure of behavioral outcome, we collect a one-day food diary at the start and finish of the study period.

Table 4. Hypothesis regarding Perceived Health Behavior Change construct.

H8 The gameplay and the increased reflection triggered by gameplay will result in an increase in 
Table 5. Measurement instruments in the study.

\begin{tabular}{|c|c|}
\hline Measurement & Instruments \\
\hline $\begin{array}{l}\text { Reflection } \\
\text { (REFL) }\end{array}$ & $\begin{array}{l}\text { The experiment used a previously validated and published self-assessment scale [21], } \\
\text { which was further studied by [22]. The original scale included three components: need for } \\
\text { reflection, engagement in reflection, and insight. Relevant questions from the scale on } \\
\text { engagement in reflection were included in the post-test questionnaire. }\end{array}$ \\
\hline $\begin{array}{l}\text { Perceived } \\
\text { persuasiveness } \\
\text { (PEPE) }\end{array}$ & $\begin{array}{l}\text { The perceived persuasiveness scale used in this experiment has been developed specifi- } \\
\text { cally for assessing BCSSs [24] and as such is a validated scale. I is a self-assessment } \\
\text { scale for assessing the impact a persuasive system. }\end{array}$ \\
\hline $\begin{array}{l}\text { Perceived } \\
\text { Health Behav- } \\
\text { ior Change } \\
\text { (PHBC) }\end{array}$ & $\begin{array}{l}\text { The instrument is based on an open-ended question presented at the end of the experiment } \\
\text { asking participants what (if any) changes they have noticed in their food choices. The } \\
\text { scale is then formed by two researchers individually categorizing all the statements into } \\
\text { five categories based on the strength and type of effect from no impact to raised aware- } \\
\text { ness, contemplation of change and actual behavioral impact. The ground-up approach of } \\
\text { the scale allows users to freely describe their experience rather than having to evaluate } \\
\text { their responses against a ready-set frame. The scale has been used earlier in [27]. }\end{array}$ \\
\hline $\begin{array}{l}\text { Rehearsal } \\
(\mathrm{REH})\end{array}$ & Collected from the game. \\
\hline $\begin{array}{l}\text { Food intake (1- } \\
\text { day food diary) }\end{array}$ & $\begin{array}{l}\text { The present study used a self-administered fruit and vegetable portion estimation form } \\
\text { [26] The questionnaire instruction included guidance for estimating a portion of vegeta- } \\
\text { bles and fruit. After guidance, portion-based estimations have been found to be realistic } \\
\text { and reliable [26]. The guidance given in this study is not directly comparable to [26], but } \\
\text { it provides a basis for comparable variables for pre- and post-test assessments. }\end{array}$ \\
\hline
\end{tabular}

\subsection{Sample selection and study procedure}

Sample selection. The sample, N=58, is one of opportunity, largely made up of university students. The participants were recruited by distributing fliers at university campus and in two shopping centers. In addition, the researchers distributed a simple advert for the experiment online in social media platforms Facebook and Twitter. Participants were offered a cinema ticket for completing the full experiment. To take part in this study participants had to be over 18 years old.

Procedure. The study consisted of starting questionnaires (background, one-day food diary), gameplay period (5 days) and final questionnaires (Reflection, Perceived Persuasiveness, Perceived Health Behavior Change and another one-day food diary). Upon sign-up participants were informed about the test setup and asked to indicate they agreed to participate. They were also informed that they could stop at any point and ask for their data to be removed. The experiment information also explained data and information security and protection of identity. A unique user ID, obtained from the mobile game, was used in all the forms in order to maintain anonymity.

The participants were instructed to play the game on five consecutive days, a minimum three sets per day, and then finally send their gaming data to the researchers by using a send button in the application. 2-3 days after sending the data, participants received the post-test questionnaire. The flexibility in sending the instruction for the final questionnaire aimed at allowing the users at least one full day after completing 
the game period before filling in the food diary but also at avoiding the reporting landing on weekend days. By encouraging working days for reporting the intention was to collect the food diaries under as similar conditions as possible.

\subsection{Materials: the game}

The game (example screens in Figure 2) in the present study was developed from the start as a highly gamified BCSS with persuasive features selected based on a Persuasive Systems Design (PSD) analysis [9]. As a means of producing the selected PSD features the game adapted the Implicit Association Test (IAT) [7] as a mechanism for a) producing feedback for the users and b) as the basis for scoring logic. The game is not intended to be an IAT in itself, but rather uses the mechanism as a means of providing instant feedback that is entirely based on the player's own reactions and responses. In other words, the score is not based on any game-originating random factors, surprises or obstacles that are not under a player's control.
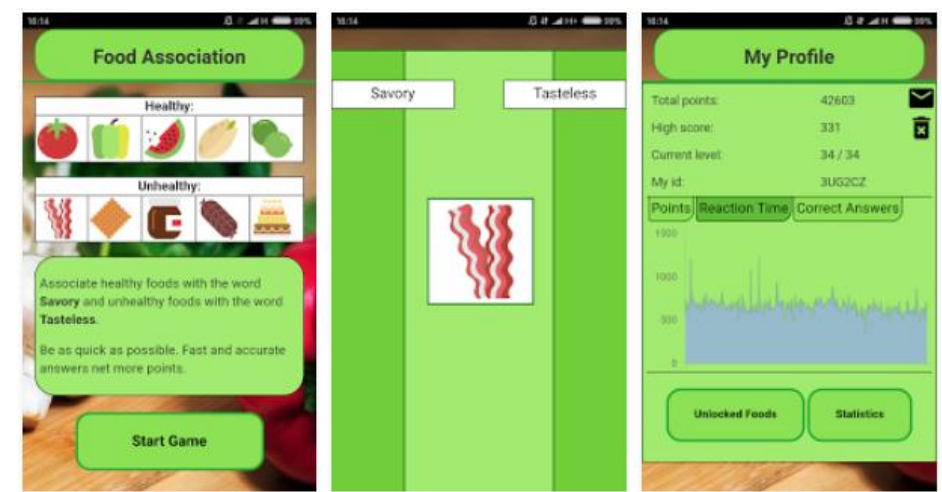

Figure 2. 'Implicity the Food Game' start screen (left), categorization task item (center) where left and right margins are the touch target, and score screen (right).

The game contains two categorization tasks. In the first task, the user has to associate healthy foods with a positive word and unhealthy foods with negative words. In the second task, positive words are associated with a healthy food and negative words with an unhealthy food. Reaction times from the point of presenting the food/word are measured. After each set of ten items a score screen is shown. Scoring was based on reaction thresholds described in [7] and [28]. Gamification elements included points, levels, and content unlocking [10].

\section{$4 \quad$ Data analysis and results}

\subsection{Sample characteristics}

Of the total sample of 58 participants 33 were female, 25 male. Average age for the whole sample was 24 years (median 23), average for females being 24 (18-35 
min/max, median 22) and for males 23 (18-33 min/max, median 23). Eight participants reported that they do not usually play any computerized games, 19 used a computer as their main gaming platform, 20 used a gaming console, and 11 reported a mobile device as their primary gaming platform. The sample size satisfies the general rule of thumb of ten times the largest number of paths directed at a particular construct [29].

\subsection{Measurement model}

Using PLS-SEM analysis the relationships between latent variables were examined. The analysis demonstrates the explained variance $\left(\mathrm{R}^{2}\right.$ values) in the latent variables, and indicates the strength ( $\beta$-values) and their statistical significance of the relationships in the model $[29,30]$. See Figure 3 for the $\mathrm{R}^{2}$-values and $\beta$-values.

Table 6. Internal consistency and indicator reliability assessment.

\begin{tabular}{llllccccc}
\hline & CA & CR & AVE & 1 & 2 & 3 & 4 & 5 \\
\hline 1. REH & 1.000 & 1.000 & 1.000 & 1.000 & & & & \\
2. Moderator (REH on & 1.000 & 1.000 & 1.000 & -0.272 & 1.000 & & & \\
REFL $>$ PEPE) & & & & & & & & \\
3. PEPE & 0.692 & 0.868 & 0.692 & 0.179 & 0.325 & 0.832 & & \\
4. PHBC & 1.000 & 1.000 & 1.000 & -0.009 & 0.080 & 0.835 & 1.000 & \\
5. REFL & 0.668 & 0.923 & 0.668 & -0.161 & 0.191 & 0.281 & 0.338 & 0.817 \\
\hline
\end{tabular}

Convergent reliability is indicated with average variance extracted (AVE) and Fornell-Larcker analysis. Square root of AVE and inner-construct correlations are shown in italics.

In terms of internal consistency, the reliability of the indicators, the convergent validity of the indicators, and the discriminant validity the measurement model adheres to recommended guidelines [29, 31, 32] (see Table 6). REH and PHBC were single indicator constructs, and while generally speaking single item constructs are not encouraged with PLS-SEM practical considerations are acceptable for example when the single items measure an observable characteristic [29].

\subsection{Structural model}

The research model (Figure 1) presented the hypotheses regarding the relationships in the model. The results of the PLS analysis are illustrated in Figure 3.

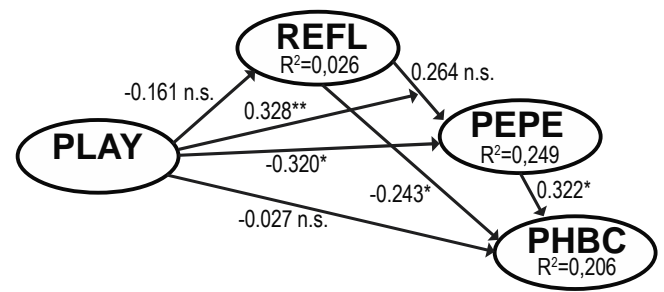

Figure 3. Structural model. $* * * \mathrm{p}<.01 ; * * \mathrm{p}<.02 ; * \mathrm{p}<.05$, n.s. for non-significant paths.

The path coefficients for the research model were obtained using parametric bootstrapping with 5000 subsamples (parallel processing, no sign changes). The constructs in the model were reflective. In the complete model we see that $21 \%$ of the variance 
in PHBC was explained by REFL and PEPE (supporting H5 and H7), and 25\% of the variance in PEPE was explained by REH (supporting $\mathrm{H} 2$ and $\mathrm{H} 4$ ); REH was a significant moderator in the relationship between REFL and PEPE. Hypotheses H1, H3 and H6 were not supported.

\subsection{Total effects and effect sizes, and predictive validity of the model}

Practical relevance of the model was determined by assessing the total effects and effect sizes (Cohen $\mathrm{f}^{2}$ ), assessing an exogenous construct's contribution to the $\mathrm{R}^{2}$ value of an endogenous latent variable (Table 7). Assessment guide values were 0.02 (small), 0.15 (medium), and 0.35 (large). In the present model there were five small, one non-relevant and one medium $\mathrm{f}^{2}$, indicating that on the whole there is some practical relevance in the model. The non-relevant $\mathrm{f}^{2}$ was $\mathrm{REH}$ to PHBC, and the medium $\mathrm{f}^{2}$ was REH as a moderator for REFL and PEPE.

Table 7. Total effects and effect sizes (non-significant not reported).

\begin{tabular}{lccc}
\hline & PEPE & PHBC & REFL \\
\hline REH & $-0.320(0.125)$ & n.s. & n.s. \\
Moderator (REH on REFL $>$ PEPE) & $0.328(0.157)$ & & \\
PEPE & & $0.322(0.113)$ & \\
PHBC & & & \\
REFL & n.s. & $0.243(0.065)$ & \\
\hline
\end{tabular}

A blindfolding procedure was used to observe the predictive validity of the model (Table 8). The Stone-Geisser cross-validated redundancy value $\left(\mathrm{Q}^{2}\right)$ above 0 is considered to indicate predictive validity of endogenous constructs. All endogenous constructs (PEPE, PHBC and REFL) demonstrate $\mathrm{Q}^{2}>0$, and thus indicate the path model's predictive relevance to each of the constructs [29].

Table 8. Predictive validity of the model: $Q^{2}$ values for endogenous constructs.

\begin{tabular}{ll}
\hline Construct & $\mathrm{Q}^{2}$ \\
\hline PEPE & 0.108 \\
PHBC & 0.122 \\
REFL & 0.011 \\
\hline
\end{tabular}

\subsection{Fruit and vegetable consumption before and after the study period}

A one-way repeated measures ANOVA was conducted to compare the effect of timing of self-reported fruit and vegetable consumption before the game play period and immediately after it (Table 9). At $\alpha$-level of .05 there was a significant effect of timing, Wilks' Lambda $=.860, \mathrm{~F}(1,57)=9.316, \mathrm{p}=.003$. The result provides indirect support for the use of PEPE and PHBC constructs in assessing the effect of system use on behavior through showing that the self-reported behavior observations are in line with the food diaries. The result supports $\mathrm{H} 8$.

Table 9. Descriptive statistics for one-way repeated measures ANOVA.

\begin{tabular}{llll}
\hline & $\mathrm{N}$ & Mean & Std.Dev. \\
\hline Pre-study vegetable consumption & 58 & 4.06 & 3.182 \\
Post-study vegetable consumption & 58 & 5.10 & 2.732 \\
\hline
\end{tabular}




\section{Discussion and conclusions}

Successful design of Behavior Change Support Systems involves selecting the right persuasive tools for the purpose [9, 34]. The forms of BCSSs are various from simple information sharing websites to interactive mobile applications to, as in in the present paper, gamified systems. Whatever the format, the basic persuasive design principles can be applied. In the game presented in this paper the selected persuasive design principles [9] were built on top of a cognitive test concept and they were implemented as gaming features, following game development principles [10].

In our study the game provided the users with an opportunity to rehearse a target behavior and also to reflect upon the rehearsal results with the help of immediate feedback. The main interest in the paper was in the effect of rehearsal on reflection leading on to perceived persuasiveness and health behavior change. Research into reflection suggests that unless the reflection leads to insight, behavior change is less likely to take place $[34,35,36]$, and dysfunctional attitudes can hinder the path from insight to personal well-being [37]. A review into use of digital games in cancer management found potential (and also challenges) in both treatment training but more notably for example when active participation or behavioral rehearsal for physical and psychosocial activity are needed [6]. The moderating role of rehearsal on the relationship between reflection and perceived persuasiveness would appear to be an element persuasive systems should consider utilizing when intending to facilitate behavior change through reflection.

What we can learn from the results is that rehearsal can amplify the effect of reflection as regards a user's perception of the persuasiveness of a system. We can also see that rehearsal alone may not be enough to result in positive behavioral outcomes: volume of game play did not affect the immediate health behavior directly in the way it did Perceived Persuasiveness. In turn, Perceived Persuasiveness had an impact on the immediate health behavior, as did Reflection. In other words, for a change to take place, both reflection and perceived persuasiveness are necessary and they can be made more effective through rehearsal. The perceived outcomes were supported in the study with the actual fruit and vegetable consumption estimates, as seen in the ANOVA on the consumption of vegetable and fruit before and after the test period.

The study naturally does not provide insights into long-term effects. The gameplay period was also relatively short (5 days) and game content was limited (only 34 levels). Further insights into the direct role of various persuasive features (such as rewards and praise) would have made for an even richer study set-up, but owing to the necessity to have participants commit to play the game for five days it was necessary to be parsimonious as regards the extent of the questionnaire batteries and demands on participant time. As existing research shows, for example [20], we can see that the relationship between implicit attitudes and behavior can be complicated. Therefore, future research directions leading on from the present study should involve the actual implicit association directions and strengths and their development after reflection. 


\section{References}

1. Ploderer, B., Reitberger, W., Oinas-Kukkonen, H. et al.: Social interaction and reflection for behaviour change Pers Ubiquit Comput 18: 1667 (2014).

2. Li, I., Dey, A. K., \& Forlizzi, J.: Understanding my data, myself: Supporting selfreflection with ubicomp technologies. Proceedings of the UbiComp 2011 International Conference on Ubiquitous Computing. New York, NY: ACM (2011).

3. Rogers, R. R.: Reflection in higher education: A concept analysis. Innovative Higher Education, 26, 37-57 (2001).

4. Kelders, S. M., Kok, R. N., Ossebaard, H. C. and Van Gemert-Pijnen, J. E.: Persuasive System Design Does Matter: A Systematic Review of Adherence to Web-Based Interventions. Journal of Medical Internet Research, 14(6) (2012).

5. Liboriussen, B.: Craft, creativity, computer games: The fusion of play and material consciousness. Philosophy \& Technology, 26(3), 273-282 (2013).

6. Ghazisaeidi, M., Safdari, R., Goodini, A., Mirzaiee, M., \& Farzi, J.: Digital games as an effective approach for cancer management: Opportunities and challenges. Journal of Education and Health Promotion, 6, 30 (2017).

7. Greenwald, A. G., McGhee, D. E., \& Schwartz, J.: Measuring individual differences in implicit cognition: the implicit association test. Journal of personality and social psychology, 74(6), 1464 (1998).

8. Oinas-Kukkonen, H.: A foundation for the study of behavior change support systems. Pers Ubiquit Comput 17(6):1223-1235 (2013).

9. Oinas-Kukkonen, H. and Harjumaa, M.: Persuasive systems design: key issues, process model, and system features. Communications of the Association for Information Systems, 24 (2009).

10. Hamari, J., and Koivisto, J.: Social motivations to use gamification: An empirical study of gamifying exercise. Proceedings of the ECIS 2013 European Conference on Information Systems (2013).

11. Huotari, K. and Hamari, J.: Defining Gamification - A Service Marketing Perspective. In proceedings of MindTrek 2012, October 3-5 (2012).

12. Werbach, K., and Hunter, D.: For the win: How game thinking can revolutionize your business. Wharton Digital Press (2012).

13. Alahäivälä, T., and Oinas-Kukkonen, H.: Understanding persuasion contexts in health gamification: a systematic analysis of gamified health behavior change support systems literature. International journal of medical informatics, 96, 62-70 (2016).

14. Kawachi, I.: It's All in the Game - The Uses of Gamification to Motivate Behavior Change. JAMA Internal Medicine 177 (11) (2017).

15. Schoech, D., Boyas, J.F., Black, B.M., and Elias-Lambert, N.: Gamification for Behavior Change: Lesons from Developing a Social, Multiuser, Web-Tablet Based Prevention Game for Youths. Journal of Technology in Human Services, 31:2, pp. 197-217 (2013).

16. King, D., Greaves, F., Exeter, C., \& Darzi, A.: "Gamification": Influencing health behaviours with games. Journal of the Royal Society of Medicine, 106(3), 76-78 (2013).

17. Kappen, D.L. and Orji, R.: Gamified and Persuasive Systems as Behavior Change Agents for Health and Wellness. In XRDS: Crossroads, The ACM Magazine for Students 24, 1: 52-55. ACM (2017).

18. Lumsden, J., Edwards, E. A., Lawrence, N. S., Coyle, D., and Munafò, M. R.: Gamification of Cognitive Assessment and Cognitive Training: A Systematic Review of Applications and Efficacy. JMIR Serious Games, 4(2), e11 (2016). 
19. Maison, D., Greenwald, A.G. and Bruin, R.: The Implicit Association Test as a Measure of Implicit Consumer Attitudes. Polish Psychological Bulletin, 32, 1-9 (2001).

20. Roefs, A., and Jansen, A.: Implicit and explicit attitudes toward high-fat foods in obesity. Journal of Abnormal Psychology, 111(3), 517-521 (2002).

21. Grant, A.M., Fraklin, J. and Langford, P.: The Self-reflection and Insight Scale: A new measure of private self-consciousness. Social Behavior and Personality: an international journal, Vol. 30, November 8, pp. 821-835(15) (2002).

22. Roberts, C., and Stark, P.: Readiness for self-directed change in professional behaviours: Factorial validation of the self-reflection and insight scale. Medical Education, 42, 10541063 (2008).

23. Crano, W. D., and Prislin, R.: Attitudes and Persuasion, Annu. Rev. Psychol 57, pp. 345-74 (2006).

24. Lehto, T., Oinas-Kukkonen, H., and Drozd, F.: Factors affecting perceived persuasiveness of a behavior change support system. Proc. of the International Conference on Information Systems (ICIS 2012) (2012).

25. Petty R.E. and Cacioppo J.T.: The elaboration likelihood model of persuasion. Advances in Experimental Social Psychology, Vol 19 (1986).

26. Cox, D.N., Anderson, A.S., Reynolds, J., McKellar, S., Lean, M.E.J., Mela, D.J.: Take five, a nutrition education intervention to increase fruit and vegetable intakes: impact on consumer choice and nutrient intakes. Br J Nutr 80(2):123-131 (1998).

27. Tikka, P. and Oinas-Kukkonen, H.: Contributing or receiving - the role of social interaction styles in persuasion over a social networking platform. Pers Ubiquit Comput 21:705721 (2017).

28. Nosek, B. A., and Banaji, M. R.: The go/no-go association task. Social cognition, 19(6), 625-666 (2001).

29. Hair, J.F., Hult, G.T.M., Ringle, C.M., Sarstedt, M.: A primer on partial least squares structural equation modeling (PLS-SEM). SAGE Publications, Los Angeles (2014).

30. Hair J.F., Ringle C.M., Sarstedt M.: PLS-SEM: indeed a silver bullet. The Journal of Marketing Theory and Practice 19(2):139-152 (2011).

31. Gefen D., Ringdon E.E., and Straub D.W.: Editor's comment: an update and extension to SEM guidelines for administrative and social science research. MIS Q 35(2):3-14 (2011).

32. Fornell C., Larker D.: Evaluating structural equation models with unobservable variables and measurement error. J Mark Res 1(18):39-50 (1981).

33. Nunally J.C, Bernstein I.: Psychometric theory. McGraw-Hill, New York (1994).

34. Fogg B.J.: Persuasive technology: using computers to change what we think and do. Morgan Kaufmann, San Francisco (2003).

35. Lyke, J. A. Insight, but not self-reflection, is related to subjective well-being. Personality and Individual Differences, 46, 66-70 (2009).

36. Halttu, K. and Oinas-Kukkonen, H.: Persuading to Reflect: Role of Reflection and Insight in Persuasive Systems Design for Physical Health. Human-Computer Interaction, Vol. 00, pp. 1- 32 (2017).

37. Stein, D., and Grant, A. M.: Disentangling the relationships among self-reflection, insight, and subjective well-being: The role of dysfunctional attitudes and core self-evaluations. The Journal of Psychology, 148, 505-522 (2014). 